\title{
(2) OPEN ACCESS \\ Burden of nosocomial COVID-19 in Wales: results from a multicentre retrospective observational study of 2508 hospitalised adults
}

\author{
Mark J Ponsford $0^{1},{ }^{1,2}$ Rhys Jefferies, ${ }^{3,4}$ Chris Davies, ${ }^{5}$ Daniel Farewell, ${ }_{1}^{6}$ \\ Ian R Humphreys ${ }^{2,7}$ Stephen Jolles, ${ }^{1}$ Sara Fairbairn, ${ }^{3,8}$ Keir Lewis, ${ }^{9,10}$ Daniel Menzies, ${ }^{11}$ \\ Amit Benjamin, ${ }^{12}$ Favas Thaivalappil, ${ }^{13}$ Chris Williams, $^{14}$ Simon M Barry ${ }^{15}$
}

\begin{abstract}
- Additional supplemental material is published online only. To view, please visit the journal online (http://dx.doi. org/10.1136/thoraxjnl-2021 216964).
\end{abstract}

For numbered affiliations see end of article.

\section{Correspondence to} Dr Mark J Ponsford, Immunodeficiency Centre for Wales, University of Wales Hospital, Cardiff, UK; ponsfordm@cardiff.ac.uk

Received 26 January 2021 Accepted 18 June 2021 Published Online First 22 July 2021

\section{Linked}

- http://dx.doi.org/10.1136/ thoraxjnl-2021-217661

Check for updates

(C) Author(s) (or their employer(s)) 2021. Re-use permitted under CC BY. Published by BMJ.

To cite: Ponsford MJ, Jefferies R, Davies C, et al. Thorax 2021;76:1246-1249.

\section{ABSTRACT}

The burden of nosocomial SARS-CoV-2 infection remains poorly defined. We report on the outcomes of 2508 adults with molecularly-confirmed SARS-CoV-2 admitted across 18 major hospitals, representing over $60 \%$ of those hospitalised across Wales between 1 March and 1 July 2020. Inpatient mortality for nosocomial infection ranged from $38 \%$ to $42 \%$, consistently higher than participants with community-acquired infection $(31 \%-35 \%)$ across a range of case definitions. Those with hospital-acquired infection were older and frailer than those infected within the community. Nosocomial diagnosis occurred a median of 30 days following admission (IQR 21-63), suggesting a window for prophylactic or postexposure interventions, alongside enhanced infection control measures.

Little is known regarding the prevalence and outcomes of in-hospital transmission of SARSCoV-2 among medical patients. ${ }^{1}$ The largest and only multicentre cohort study to date reported outcomes in 1564 patients admitted with confirmed SARS CoV-2 infection across 11 hospitals. ${ }^{2}$ Mortality in the nosocomial group appeared comparable to those with likely community-acquired infection $\left(27.0 \%\right.$ and $27.2 \%$, respectively). ${ }^{2}$ This study was conducted early in the pandemic course, meaning reliable estimates of the true impact of hospitalacquired COVID-19 infection remain hampered by a paucity of publicly available data at national and regional levels. ${ }^{3}$ Here, we update assessment of the relative burden of community-acquired and nosocomial-acquired SARS-CoV-2 infection, using anonymised patient-level and hospital-level data collected via the National Pathway for Managing COVID-19 Infections in Secondary Care in Wales initiative (www.covid-19hospitalguideline.wales. nhs.uk).

The methods and data sources relating to this work are described in detail elsewhere. ${ }^{4}$ Briefly, positive SARS-CoV-2 PCR results recorded between 1 March 2020 and 1 July 2020 in adults with a recorded hospital admission were identified for retrospective notes review. Local clinical teams across 18 centres (online supplemental file S1) performed data entry using a standardised online tool. Mandatory fields included dates of PCR sampling, admission and discharge, age, sex, comorbidity count and outcome (death or discharge). Supplementary fields included Welsh
Index of Multiple of Deprivation (WIMD) and preadmission Clinical Frailty Scale (CFS).

The primary outcome was all-cause mortality, grouped by probable origin of SARS-CoV-2 infection based on (1) clinician-recorded source and (2) standardised case definitions (online supplemental file S2). Time-to-event analysis used time in hospital following a positive PCR test, to avoid introducing survivorship bias. All analyses were performed using R and GraphPad Prism.

We identified 6005 SARS-CoV-2-positive results with a location in hospital, taken between 1 March 2020 and 1 July 2020 inclusive, of which 4112 were individual cases. Clinical information was obtained from $2584 / 4112$ individuals (63\%). A total of 76 individuals were excluded due to missing core data fields or initial PCR sampling date exceeding admission period by 31 days (online supplemental file S3). This left 2508 case records, representing approximately $61 \%$ of the total adult population hospitalised with COVID-19 within Wales. Admission features are summarised in table 1 . The cohort had a median age of 74 years (IQR 62.5-85.5), of whom $54.3 \%$ were men and $45.7 \%$ were women. Individuals from the most-deprived WIMD quartile were over-represented relative to those in the least deprived quartile $\left(31.2 \%\right.$ vs $18.7 \%, \chi^{2}$ test: $\mathrm{p}<0.0001)$.

Clinician-defined admission source was available in 2354 cases (93.9\%). Hospital-acquired COVID-19 was documented in 433 cases $(17.3 \%$ of cohort, $37.8 \%$ mortality), comparable to mortality in cases presenting by ambulance (553/1359, 40.7\%). Walk-in and GP referrals together accounted for $20.4 \%$ of the cohort and had the lowest inpatient mortality rate $(17.1 \%-23.6 \%)$. The small number of patients admitted from care or nursing homes showed the highest inpatient mortality rate $(23 / 50,46.0 \%)$.

We next applied a standardised definition for nosocomial COVID-19, based on the interval between admission and diagnostic testing exceeding 14 days, identifying 411 cases $(16.4 \%$ of cohort, consistent with previous reports). ${ }^{2}$ Community-acquired cases constituted the majority ( $n=1604,64.0 \%)$, defined by PCR sampling preceding or within 5 days of admission. ${ }^{2}$ Monthly prevalence estimates are shown in online supplemental file S4. Overall, $39.2 \%$ of patients with nosocomial-infection died, compared with $31.7 \%$ with community-acquired infection. This proved consistent across the 


\begin{tabular}{|c|c|c|c|}
\hline Variable & Died (\%) & Discharged (\%) & Total (\%) \\
\hline Admission hospital & & & 2508 \\
\hline A & $174(40.6)$ & $255(59.4)$ & $429(17.1)$ \\
\hline B & $165(38.9)$ & $259(61.1)$ & $424(16.9)$ \\
\hline C & $96(39.8)$ & $145(60.2)$ & $241(9.6)$ \\
\hline D & $78(32.9)$ & $159(67.1)$ & $237(9.4)$ \\
\hline $\mathrm{E}$ & $97(42.9)$ & $129(57.1)$ & $226(9.0)$ \\
\hline$F$ & $46(27.1)$ & $124(72.9)$ & $170(6.8)$ \\
\hline G & $35(22.4)$ & $121(77.6)$ & $156(6.2)$ \\
\hline H & $48(33.3)$ & $96(66.7)$ & $144(5.7)$ \\
\hline I & $19(22.6)$ & 65 (77.4) & $84(3.3)$ \\
\hline J & $22(27.2)$ & $59(72.8)$ & $81(3.2)$ \\
\hline $\mathrm{K}$ & $35(43.2)$ & $46(56.8)$ & $81(3.2)$ \\
\hline L & $24(32.0)$ & $51(68.0)$ & $75(3.0)$ \\
\hline M & $14(21.2)$ & $52(78.8)$ & $66(2.6)$ \\
\hline $\mathrm{N}$ & $24(38.7)$ & $38(61.3)$ & $62(2.5)$ \\
\hline 0 & $6(25.0)$ & $18(75.0)$ & $24(1.0)$ \\
\hline $\mathrm{P}^{*}$ & $2(25.0)$ & $6(75.0)$ & $8(0.3)$ \\
\hline
\end{tabular}

Age group (years)

\begin{tabular}{|c|c|c|c|}
\hline$<65$ & $115(14.7)$ & $667(85.3)$ & $782(31.2)$ \\
\hline $65-75$ & $305(44.4)$ & $382(55.6)$ & $687(27.4)$ \\
\hline $75-85$ & $273(50.6)$ & 267 (49.4) & $540(21.5)$ \\
\hline$>85$ & $192(38.5)$ & $307(61.5)$ & $499(19.9)$ \\
\hline \multicolumn{4}{|l|}{ Sex } \\
\hline Female & 377 (32.9) & $768(67.1)$ & $1145(45.7)$ \\
\hline Male & $508(37.3)$ & $855(62.7)$ & $1363(54.3)$ \\
\hline $\begin{array}{l}\text { Median comorbidity count } \\
\text { (IQR) }\end{array}$ & $3(2-4)$ & $2(0.5-3.5)$ & $2(0.5-3.5)$ \\
\hline \multicolumn{4}{|l|}{ Supplementary fields } \\
\hline \multicolumn{4}{|l|}{ WIMD† } \\
\hline Q1-most deprived & $265(33.9)$ & $517(66.2)$ & $782(31.2)$ \\
\hline Q2 & $251(38.0)$ & $409(62.0)$ & $660(26.3)$ \\
\hline Q3 & $163(34.5)$ & $310(65.5)$ & $473(18.9)$ \\
\hline Q4-least deprived & $168(35.7)$ & $302(64.3)$ & $470(18.7)$ \\
\hline WIMD unrecorded & $38(30.9)$ & $85(69.1)$ & $123(4.9)$ \\
\hline \multicolumn{4}{|l|}{ CFS score } \\
\hline 1-very fit & $21(12.8)$ & $143(87.2)$ & $164(6.5)$ \\
\hline 2 -fit & $32(16.1)$ & $167(83.9)$ & $199(7.9)$ \\
\hline 3-managing well & $47(27.3)$ & $125(72.7)$ & $172(6.9)$ \\
\hline 4-vulnerable & $63(39.9)$ & $95(60.1)$ & $158(6.3)$ \\
\hline 5-mildly frail & $70(52.6)$ & $63(47.4)$ & $133(5.3)$ \\
\hline 6-frail & $117(49.6)$ & $119(50.4)$ & $236(9.4)$ \\
\hline 7—severely frail & $87(50.0)$ & $87(50.0)$ & $174(6.9)$ \\
\hline 8 -very severely frail & $36(62.1)$ & $22(37.9)$ & $58(2.3)$ \\
\hline 9-terminally ill & $7(63.6)$ & $4(36.4)$ & $11(0.4)$ \\
\hline CFS score unrecorded & $405(33.7)$ & $798(66.3)$ & $1203(48.0)$ \\
\hline \multicolumn{4}{|l|}{ Ceiling of care } \\
\hline Intensive care & $102(30.0)$ & $238(70.0)$ & $340(13.6)$ \\
\hline Ward (CPAP) & $98(42.2)$ & $134(57.8)$ & $232(9.2)$ \\
\hline Ward (no CPAP) & $572(41.2)$ & $817(58.8)$ & $1389(55.4)$ \\
\hline
\end{tabular}

Continued

\begin{tabular}{llll}
\hline Table 1 Continued & & & \\
\hline Variable & Died (\%) & Discharged (\%) & Total (\%) \\
\hline $\begin{array}{l}\text { Ceiling of care } \\
\text { unrecorded }\end{array}$ & $113(20.7)$ & $434(79.3)$ & $547(21.8)$ \\
\hline
\end{tabular}

${ }^{*}$ Represents three combined centres ( $<5$ patients each). tWIMD, $1=$ =most deprived, 1909=least deprived.

CFS, Clinical Frailty Scale; CPAP, continuous positive airway pressure; WIMD, Welsh Index of Multiple Deprivation.

majority of admission sites (figure 1). Using a random effects model, we found that the relative risk of mortality in patients with nosocomial-acquired COVID-19, averaged across sites, was 1.24 times that of community-acquired infection $(95 \% \mathrm{CI} 1.06$ to $1.42, \mathrm{p}=0.0047$; online supplemental file S5). The median time from diagnostic sampling to discharge in patients with nosocomial infection was 17 days (IQR 7-38), compared with 7 days (IQR 3-15) in community-acquired COVID-19 cases. Nosocomial-infection cases had an increased cumulative incidence of inpatient mortality, when accounting for the competing risk of discharge (figure 2). Half of those with a nosocomial diagnosis had been admitted for at least 30 days prior to testing (IQR 21-63), with 48 admitted for over 100 days. Mortality by age group appeared similar for both nosocomial and community COVID-19 cases, but with a greater proportion of elderly individuals within the nosocomial COVID-19 group (online supplemental file S6).

As $95 \%$ of individuals display symptoms between 2.5 and 11.5 days of exposure, ${ }^{5}$ the above definition presents a conservative estimate of the burden of nosocomial infection. We extended analysis by varying the diagnostic interval across this range, thereby encompassing wider case definitions in use by UK Public Health agencies. Inpatient mortality rates for nosocomial COVID-19 ranged from $37.8 \%$ to $42.3 \%$ and remained greater than that for community-acquired infection $(31.4 \%-34.7 \%$, online supplemental files S7 and S8). By contrast, varying the case definition resulted in significant changes in nosocomial caseload and deaths. Applying the Public Health England definition (diagnosis $>7$ days following admission) identified 7247 (28.9\%) admissions and 300 deaths (41.4\% mortality). This rose to 827 cases $(33.0 \%)$ and 341 deaths ( $41.2 \%$ mortality), when a 5-day interval between PCR testing and admission was used. ${ }^{6}$

Finally, we investigated the vulnerability of individuals with nosocomial COVID-19. To minimise selection bias inherent with previous case definitions requiring a prolonged preinfection admission, ${ }^{2}$ we considered a diagnostic interval of 2 days postadmission, commonly used for hospital-acquired bacterial pneumonia. ${ }^{7}$ Here, nosocomial-acquired cases had a median CFS score of 5 (IQR 4-7), compared with 3 (IQR 2-6) in communityacquired cases (online supplemental file S9). Marked differences in multimorbidity were evident, with $35.0 \%$ of nosocomial cases having at least four comorbidities compared with $26.7 \%$ of community-acquired patients.

Our findings expose the hitherto underestimated vulnerability and impact of nosocomial infection with SARS-CoV-2. Many potential mechanisms may underlie these observations, including the advanced age and frailty of patients who remain admitted to the hospital during the pandemic. ${ }^{28}$ These both predispose to severe disease ${ }^{9}$ and implicate personal care requirement as a causal link to exposure. ${ }^{10}$

Study strengths include the high proportion of patients hospitalised with COVID-19 (over 60\%) across Wales with available core data, comparing favourably to similar reports. ${ }^{2} 9$ We employed simple but robust statistical 


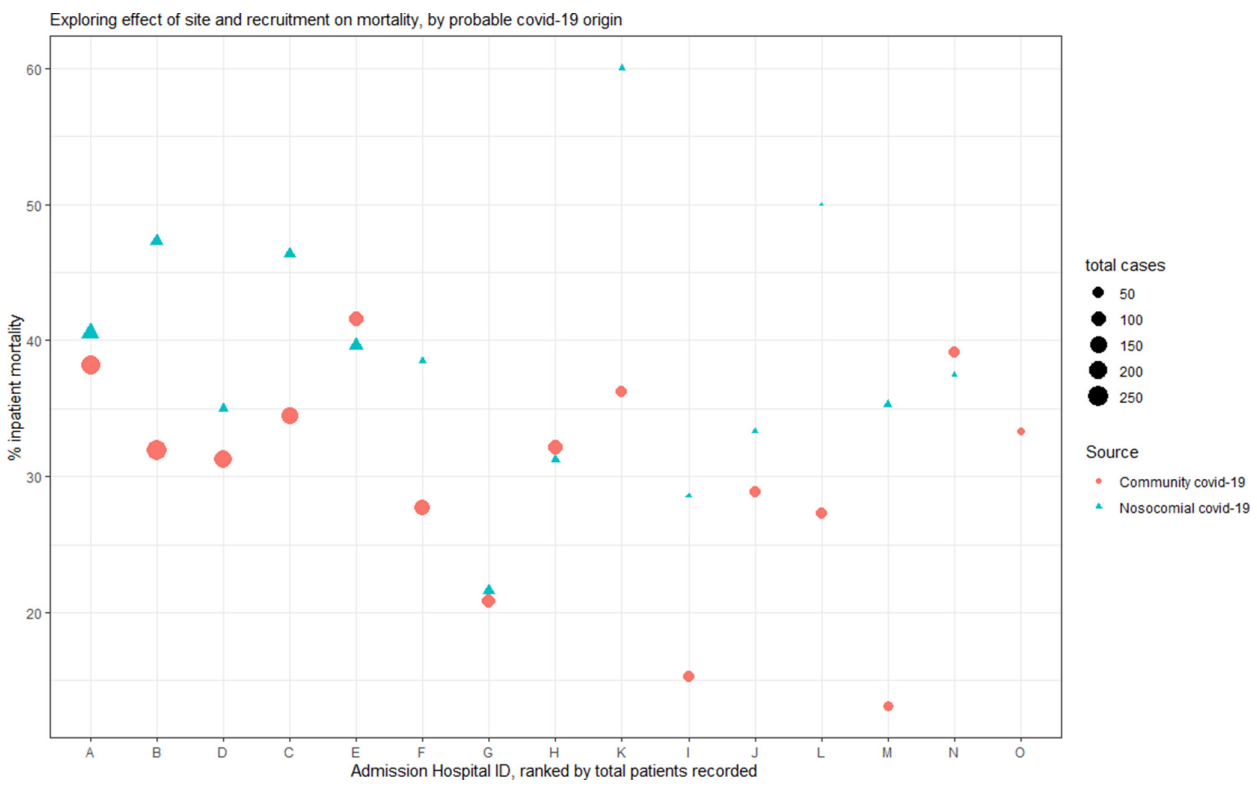

Figure 1 Inpatient mortality rates by admission hospital sites scatter plot showing inpatient mortality rates for patients with community-acquired COVID-19 (circles) and nosocomial COVID-19 (triangles) by individual sites, with hospitals arranged by decreasing overall case load are plotted from the left. for $11 / 15$ sites, inpatient mortality rates for nosocomial cases exceeds that of community acquired cases. Individual sites with fewer than five cases were excluded from analysis.

methodology, acknowledging the competing risks of discharge and death and multiple case definitions. This is relevant to interpretation of publicly reported figures. For instance, defining nosocomial cases based on the median 5-day incubation period ${ }^{5}$ identified $14.2 \%$ additional cases and $13.7 \%$ more deaths than a commonly used 7-day threshold. This suggests the burden of nosocomial COVID-19 may be significantly under-reported, which has major public health implications for infection control policy globally, particularly given the rapid spread of more infectious and severe SARSCoV-2 variants.

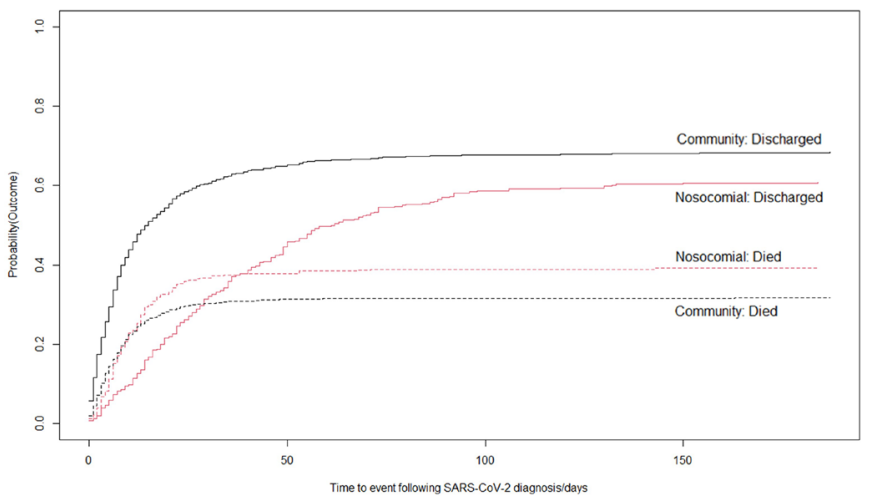

Figure 2 Competing risk analysis plot of nosocomial and community infection outcomes of patients with COVID-19. Time to event analysis cumulative incidence analysis for the competing risks of discharge and diagnosis, using the time from SARS-CoV-2 diagnosis. COVID-19 origin is assigned by the commonly used case definition, as outlined by Carter et $\mathrm{al}^{2}{ }^{2}$ nosocomial and community-acquired COVID-19 as labelled. Dotted lines: cumulative incidence of death, continuous lines: cumulative incidence of discharge on probability scale. To deal with potential survivorship bias introduced by including communitydiagnoses tested prior to admission (who cannot reach discharge or death until admission), day 0 was defined as the more recent of day of admission or date of first positive diagnostic SARS-CoV-2 testing.
Our study also has limitations, including its retrospective nature. Although sites retrieved notes at random, we cannot fully exclude risk of ascertainment bias. As the total number of patients at risk of infection was unknown, we cannot infer the risk of acquiring SARS-CoV-2 within the hospital. Similarly, we did not collect data on recent hospitalisations, and it is possible that nosocomial COVID-19 cases have been classified as community. We also recognise our findings represent crude inpatient mortality rate estimates, based on all-cause mortality. Future studies using national linked datasets including genomic analysis and estimating excess mortality are suggested.

In conclusion, we performed a national service evaluation to document the burden of nosocomial-SARS-CoV-2 infection during and following the first wave in Wales. We found many of those dying with probable hospital-acquired COVID-19 had been in the hospital for at least a month prior to exposure. We suggest this highlights an opportunity for pre-exposure and early postexposure prophylactic measures, including inpatient vaccination and clinical trial enrolment. $^{1112}$

This work was presented to the Welsh Technical Advisory Group and Directors of Nursing Group, contributing to a recommendation to ministers supporting vaccination of inpatients without a diagnosis of COVID-19 within priority groups and those being admitted for a planned procedure at increased risk.

\section{Author affiliations}

${ }^{1}$ Immunodeficiency Centre for Wales, University of Wales Hospital, Cardiff, UK ${ }^{2}$ Division Infection, Immunity, and Inflammation, School of Medicine, Cardiff University, Cardiff, UK

${ }^{3}$ Respiratory Health Implementation Group, NHS Wales Collaborative, Swansea, UK

${ }^{4}$ Swansea University Medical School, Institute of Life Science, Swansea University,

Swansea, UK

${ }^{5}$ The Institute of Clinical Science and Technology, Cardiff, UK

${ }^{6}$ Division of Population Medicine, School of Medicine, Cardiff University, Cardiff, UK

${ }^{7}$ Systems Immunity Research Institute, Cardiff University, Cardiff, UK

${ }^{8}$ Department of Respiratory Medicine, Aneurin Bevan Health Board, Newport, UK 
${ }^{9}$ Department of Medicine, Prince Philip Hospital, Hywel Dda University Health Board, Carmarthen, UK

${ }^{10}$ College of Human and Health Sciences, Swansea University, Swansea, UK

${ }^{11}$ Department of Respiratory Medicine, Betsi Cadwaladr University Health Board, Bangor, UK

${ }^{12}$ Department of Respiratory Medicine, Cwm Taf University Health Board, Abercynon, UK

${ }^{13}$ Department of Respiratory Medicine, Swansea Bay University Health Board, Port Talbot, UK

${ }^{14}$ Communicable Disease Surveillance Centre, Public Health Wales, Cardiff, UK

${ }^{15}$ Department of Respiratory Medicine, Cardiff and Vale University Health Board, Cardiff, UK

Twitter Mark J Ponsford @WCATImmunology and lan R Humphreys @irhumphreys

Acknowledgements This work uses data collected by the NHS and Public Health Wales as part of the routine care and support for patients admitted with COVID-19. We are extremely grateful to the front-line NHS junior doctors for their commitment to inputting data from across Wales. Anonymised data handling and storage was supported by The Institute of Clinical Science and Technology, and we thank Gareth Davies and Bea Addison from Institute of Clinical Science and Technology for database management and coordination of data collection, and Dr Tom J C Ward for statistical guidance and careful reading of the manuscript.

Contributors SB conceived the study and worked with RJ and CD to create the COVID-19 guideline and the database. CW provided data on behalf of Public Health Wales. MJP led the data analysis supported by DF and wrote the first draft supervised by SB, DF, SJ, and IH. CD leads the Institute for Clinical Science and Technology, which created the digital interventions. MJP, RJ, CD and SB have verified the underlying data and act as guarantors. All authors contributed scientific and clinical guidance regarding study design and data collection, and reviewed the final draft. The corresponding author attested that all listed authors met authorship criteria and that no others meeting the criteria had been omitted.

Funding The Welsh Government funds the Respiratory Health Implementation Group (RHIG) of which SB is the lead and RJ the programme manager. RHIG fund the Institute for Clinical Science and Technology (ICST) which created the digital interventions including the Welsh Hospital covid-19COVID-19 guideline and the on-line data collection tool. This work was partly funded by UKRI/NIHR through the UK Coronavirus Immunology Consortium (UK-CIC). MJP is supported by the Welsh Clinical Academic Training (WCAT) programme and a Career Development Award from the Association of Clinical Pathologists and is a participant in the NIH Graduate Partnership Programme. IH is a Wellcome Trust Senior Research Fellow in Basic Biomedical Sciences.

Competing interests None declared.

Patient consent for publication Not required.

Ethics approval This work was conducted as service evaluation. A formal waiver for ethical approval was received from Approvals Officer Dr Beresford (Welsh REC2). Requirement for ethical approval for this service evaluation was formally waived.
Provenance and peer review Not commissioned; externally peer reviewed.

Open access This is an open access article distributed in accordance with the Creative Commons Attribution 4.0 Unported (CC BY 4.0) license, which permits others to copy, redistribute, remix, transform and build upon this work for any purpose, provided the original work is properly cited, a link to the licence is given, and indication of whether changes were made. See: https://creativecommons.org/ licenses/by/4.0/.

\section{ORCID iD}

Mark J Ponsford http://orcid.org/0000-0002-0236-1059

\section{REFERENCES}

1 NHS England and NHS Improvement. Minimising nosocomial infections in the NHS, 2020. Available: https://www.england.nhs.uk/coronavirus/wp-content/uploads/sites/ 52/2020/06/C0586-minimising-nosocomial-infections-in-the-nhs.pdf; [Accessed 02-December-2020].

2 Carter B, Collins JT, Barlow-Pay F, et al. Nosocomial COVID-19 infection: examining the risk of mortality. The COPE-Nosocomial study (COVID in older people). J Hosp Infect 2020;106:376-84.

3 Healthcare Safety Investigation Branch (HSIB). COVID-19 transmission in hospitals: management of the risk - a prospective safety investigation. independent report by the healthcare safety investigation branch 12020/018, 2020. Available: https://www hsib.org.uk/documents/257/hsib-report-covid-19-transmission-hospitals.pdf

4 Ponsford MJ, Jefferies R, Davies C. The burden of nosocomial covid-19: results from the Wales multi-centre retrospective observational study of 2518 hospitalised adults. medRxiv2021:2021.01.18.21249433.

5 Lauer SA, Grantz KH, Bi Q, et al. The incubation period of coronavirus disease 2019 (COVID-19) from publicly reported confirmed cases: estimation and application. Ann Intern Med 2020;172:577-82.

6 Gupta RK, Marks M, Samuels THA. Systematic evaluation and external validation of 22 prognostic models among hospitalised adults with COVID-19: an observational cohort study. medRxiv2020:2020.07.24.20149815.

7 Kalil AC, Metersky ML, Klompas M, et al. Management of adults with hospitalacquired and ventilator-associated pneumonia: 2016 clinical practice guidelines by the infectious diseases Society of America and the American thoracic Society. Clin Infect Dis 2016:63:e61-111.

8 Hewitt J, Carter B, Vilches-Moraga A, et al. The effect of frailty on survival in patients with COVID-19 (cope): a multicentre, European, observational cohort study. Lancet Public Health 2020;5:e444-51.

9 Docherty AB, Harrison EM, Green CA, et al. Features of 20133 UK patients in hospital with covid-19 using the ISARIC WHO Clinical Characterisation Protocol: prospective observational cohort study. BMJ 2020;369:m1985.

10 Lucey M, Macori G, Mullane N, et al. Whole-Genome sequencing to track severe acute respiratory syndrome coronavirus 2 (SARS-CoV-2) transmission in nosocomial outbreaks. Clin Infect Dis 2021;72:e727-35.

11 Bratzler DW, Houck PM, Jiang $H$, et al. Failure to vaccinate Medicare inpatients: a missed opportunity. Arch Intern Med 2002;162:2349-56.

12 Libster R, Pérez Marc G, Wappner D, et al. Early high-titer plasma therapy to prevent severe Covid-19 in older adults. N Engl J Med 2021;384:610-8. 